\title{
EXAMPLES OF DOMAINS WITH NON-COMPACT AUTOMORPHISM GROUPS
}

\begin{abstract}
Siqi Fu, A. V. Isaev, and S. G. Krantz
A вstract. We give an example of a bounded, pseudoconvex, circular domain in $\mathbb{C}^{n}$ for any $n \geq 3$ with smooth real-analytic boundary and noncompact automorphism group, which is not biholomorphically equivalent to any Reinhardt domain. We also give an analogous example in $\mathbb{C}^{2}$, where the domain is bounded, non-pseudoconvex and such that the boundary is smooth real-analytic at all points except one and is $C^{1, \alpha}$-smooth at the exceptional point.
\end{abstract}

Let $D$ be a bounded or, more generally, a hyperbolic domain in $\mathbb{C}^{n}$. Denote by $\operatorname{Aut}(D)$ the group of biholomorphic self-mappings of $D$. The group $\operatorname{Aut}(D)$, with the topology given by uniform convergence on compact subsets of $D$, is in fact a Lie group [Kob].

A domain $D$ is called Reinhardt if the standard action of the $n$-dimensional torus $\mathbb{T}^{n}$ on $\mathbb{C}^{n}$,

$$
z_{j} \mapsto e^{i \phi_{j}} z_{j}, \quad \phi_{j} \in \mathbb{R}, \quad j=1, \ldots, n,
$$

leaves $D$ invariant. For certain classes of domains with non-compact automorphism groups, Reinhardt domains serve as standard models up to biholomorphic equivalence (see e.g. [R], [W], [BP1], [BP2], [GK1], [Kod]).

It is an intriguing question whether any domain in $\mathbb{C}^{n}$ with non-compact automorphism group and satisfying some natural geometric conditions is biholomorphically equivalent to a Reinhardt domain. The history of the study of domains with non-compact automorphism groups shows that there were expectations that the answer to this question would be positive (see

Received February 23, 1996.

Mathematics Subject Classification: 32A07, 32H05, 32M05.

This work was completed while the second author was an Alexander von Humboldt Fellow at the University of Wuppertal.

Research at MSRI by the third author was supported in part by NSF Grant DMS9022140 . 
[Kra]). In this note we give examples that show that the answer is in fact negative.

While the domain that we shall consider in Theorem 1 below has already been noted in the literature [BP2], [BP3] it has never been proved that this domain is not biholomorphically equivalent to a Reinhardt domain. Note that this domain is circular, i.e. it is invariant under the special rotations

$$
z_{j} \mapsto e^{i \phi} z_{j}, \quad \phi \in \mathbb{R}, \quad j=1, \ldots, n .
$$

Our first result is the following

Theorem 1. There exists a bounded, pseudoconvex, circular domain $\Omega \subset$ $\mathbb{C}^{3}$ with smooth real-analytic boundary and non-compact automorphism group, which is not biholomorphically equivalent to any Reinhardt domain.

Proof. Consider the domain

$$
\Omega=\left\{\left|z_{1}\right|^{2}+\left|z_{2}\right|^{4}+\left|z_{3}\right|^{4}+\left(\overline{z_{2}} z_{3}+\overline{z_{3}} z_{2}\right)^{2}<1\right\} .
$$

The domain $\Omega$ is invariant under the action of the two-dimensional torus $\mathbb{T}^{2}$

$$
\begin{array}{lll}
z_{1} \mapsto e^{i \phi_{1}} z_{1}, & \phi_{1} \in \mathbb{R}, & \\
z_{j} \mapsto e^{i \phi_{2}} z_{j}, & \phi_{2} \in \mathbb{R}, \quad j=2,3,
\end{array}
$$

and therefore is circular. It is also a pseudoconvex, bounded domain with smooth real-analytic boundary. The automorphism group Aut $(\Omega)$ is noncompact since it contains the following subgroup

$$
z_{1} \mapsto \frac{z_{1}-a}{1-\bar{a} z_{1}}, \quad z_{2} \mapsto \frac{\left(1-|a|^{2}\right)^{\frac{1}{4}} z_{2}}{\left(1-\bar{a} z_{1}\right)^{\frac{1}{2}}}, \quad z_{3} \mapsto \frac{\left(1-|a|^{2}\right)^{\frac{1}{4}} z_{3}}{\left(1-\bar{a} z_{1}\right)^{\frac{1}{2}}}
$$

for a complex parameter $a$ with $|a|<1$.

We are now going to explicitly determine $\operatorname{Aut}(\Omega)$. Let $F=\left(f_{1}, f_{2}, f_{3}\right)$ be an automorphism of $\Omega$. Then, since $\Omega$ is bounded, pseudoconvex and has real-analytic boundary, $F$ extends smoothly to $\bar{\Omega}$ [BL]. Therefore, $F$ must preserve the rank of the Levi form $\mathcal{L}_{\partial \Omega}(q)$ of $\partial \Omega$ at every $q \in \partial \Omega$. The only points where $\mathcal{L}_{\partial \Omega} \equiv 0$ are those of the form $\left(e^{i \alpha}, 0,0\right), \alpha \in \mathbb{R}$. These points must be preserved by $F$. This observation implies that $f_{j}\left(e^{i \alpha}, 0,0\right)=0$ for all $\alpha \in \mathbb{R}, j=2,3$. Restricting $f_{2}, f_{3}$ to the unit disc $\Omega \cap\left\{z_{2}=z_{3}=0\right\}$, we see that $f_{j}\left(z_{1}, 0,0\right)=0$ for all $\left|z_{1}\right| \leq 1, j=2,3$. Therefore, $F(0)=(b, 0,0)$ for some $|b|<1$. Taking the composition of $F$ and the automorphism $G$ of the form (1) with $a=b$, we find that the mapping $G \circ F$ preserves the origin. Since $\Omega$ is circular, it follows from a theorem of H. Cartan $[C]$ that 
$G \circ F$ must be linear. Therefore any automorphism of $\Omega$ is the composition of a linear automorphism and an automorphism of the form (1).

The above argument also shows that any linear automorphism of $\Omega$ can be written as

$$
z_{1} \mapsto e^{i \phi_{1}} z_{1}, \quad z_{2} \mapsto a z_{2}+b z_{3}, \quad z_{3} \mapsto c z_{3}+d z_{3},
$$

where $\phi_{1} \in \mathbb{R}, a, b, c, d \in \mathbb{C}$, and the transformation in the variables $\left(z_{2}, z_{3}\right)$ is an automorphism of the section $\Omega \cap\left\{z_{1}=0\right\}$. Further, since the only points of $\partial \Omega$ where $\operatorname{rank} \mathcal{L}_{\partial \Omega}=1$ are those of the form $\left(z_{1}, w, \pm w\right)$ with $w \neq 0$ and since automorphisms of $\Omega$ preserve such points, it follows that any linear automorphism of $\Omega$ is in fact given by

$$
z_{1} \mapsto e^{i \phi_{1}} z_{1}, \quad z_{2} \mapsto e^{i \phi_{2}} z_{\sigma(2)}, \quad z_{3} \mapsto \pm e^{i \phi_{2}} z_{\sigma(3)},
$$

where $\phi_{1}, \phi_{2} \in \mathbb{R}$, and $\sigma$ is a permutation of the set $\{2,3\}$.

The preceding description of $\operatorname{Aut}(\Omega)$ implies that $\operatorname{dim} \operatorname{Aut}(\Omega)=4$. That is to say, each of the four connected components of $\operatorname{Aut}(\Omega)$ is parametrized by the point $a$ from the unit disc and by the rotation parameters $\phi_{1}, \phi_{2}$.

Suppose now that $\Omega$ is biholomorphically equivalent to a Reinhardt domain $D \subset \mathbb{C}^{3}$. Since $\Omega$ is bounded, it follows that $D$ is hyperbolic. It follows from $[\mathrm{Kru}]$ that any hyperbolic Reinhardt domain $G \subset \mathbb{C}^{n}$ can be biholomorphically mapped onto its normalized form $\tilde{G}$ for which the identity component $\operatorname{Aut}_{0}(\tilde{G})$ of $\operatorname{Aut}(\tilde{G})$ is described as follows. There exist integers $0 \leq s \leq t \leq p \leq n$ and $n_{i} \geq 1, i=1, \ldots, p$, with $\sum_{i=1}^{p} n_{i}=n$, and real numbers $\alpha_{i}^{k}, i=1, \ldots, s, k=t+1, \ldots, p$, and $\beta_{j}^{k}, j=s+1, \ldots, t$, $k=t+1, \ldots, p$ such that if we set $z^{i}=\left(z_{n_{1}+\cdots+n_{i-1}+1}, \ldots, z_{n_{1}+\cdots+n_{i}}\right)$, $i=1, \ldots, p$, then $\operatorname{Aut}_{0}(\tilde{G})$ is given by the mappings

$$
\begin{aligned}
& z^{i} \mapsto \frac{A^{i} z^{i}+b^{i}}{c^{i} z^{i}+d^{i}}, \quad i=1, \ldots, s, \\
& z^{j} \mapsto B^{j} z^{j}+e^{j}, \quad j=s+1, \ldots, t, \\
& z^{k} \mapsto C^{k} \frac{\prod_{j=s+1}^{t} \exp \left(-\beta_{j}^{k}\left(2{\overline{e^{j}}}^{T} B^{j} z^{j}+\left|e^{j}\right|^{2}\right)\right) z^{k}}{\prod_{i=1}^{s}\left(c^{i} \cdot z^{i}+d^{i}\right)^{2 \alpha_{i}^{k}}}, \\
& k=t+1, \ldots, p,
\end{aligned}
$$

where

$$
\begin{aligned}
& \left(\begin{array}{cc}
A^{i} & b^{i} \\
c^{i} & d^{i}
\end{array}\right) \in S U\left(n_{i}, 1\right), \quad i=1, \ldots, s, \\
& B^{j} \in U\left(n_{j}\right), \quad e^{j} \in \mathbb{C}^{n_{j}}, \quad j=s+1, \ldots, t, \\
& C^{k} \in U\left(n_{k}\right), \quad k=t+1, \ldots, p .
\end{aligned}
$$


The normalized form $\tilde{G}$ is written as

$$
\begin{aligned}
G=\left\{\left|z^{1}\right|\right. & <1, \ldots,\left|z^{s}\right|<1, \\
& \left(\frac{z^{t+1}}{\prod_{i=1}^{s}\left(1-\left|z^{i}\right|^{2}\right)^{\alpha_{i}^{t+1}} \prod_{j=s+1}^{t} \exp \left(-\beta_{j}^{t+1}\left|z^{j}\right|^{2}\right)}, \ldots,\right. \\
& \left.\left.\frac{z^{p}}{\prod_{i=1}^{s}\left(1-\left|z^{i}\right|^{2}\right)^{\alpha_{i}^{p}} \prod_{j=s+1}^{t} \exp \left(-\beta_{j}^{p}\left|z^{j}\right|^{2}\right)}\right) \in \tilde{G}_{1}\right\},
\end{aligned}
$$

where $\tilde{G}_{1}:=\tilde{G} \bigcap\left\{z^{i}=0, i=1, \ldots, t\right\}$ is a hyperbolic Reinhardt domain in $\mathbb{C}^{n_{t+1}} \times \cdots \times \mathbb{C}^{n_{p}}$.

It is now easy to see that, for any hyperbolic Reinhardt domain $D \subset \mathbb{C}^{3}$ written in a normilized form $\tilde{D}, \operatorname{Aut}_{0}(\tilde{D})$ given by formulas (2) cannot have dimension equal to 4 .

This completes the proof.

Remark. The theorem can be easily extended to $\mathbb{C}^{n}$ for any $n \geq 3$ (just replace $\left|z_{1}\right|^{2}$ in the defining function of $\Omega$ by $\sum_{j=1}^{n-2}\left|z_{j}\right|^{2}, z_{2}$ by $z_{n-1}, z_{3}$ by $\left.z_{n}\right)$.

There is considerable evidence that, in complex dimension two, an example such as that constructed in Theorem 1 does not exist. Certainly the example provided above depends on the decoupling, in the domain $\Omega$, of the variables $z_{2}, z_{3}$ from the variable $z_{1}$. Such decoupling is not possible when the dimension is only two.

The work of Bedford and Pinchuk (see [BP2] and references therein) suggests that the only smoothly bounded domains in $\mathbb{C}^{2}$ with non-compact automorphism groups are (up to biholomorphic equivalence) the complex ellipsoids

$$
\Omega_{m}=\left\{\left(z_{1}, z_{2}\right) \in \mathbb{C}^{2}:\left|z_{1}\right|^{2}+\left|z_{2}\right|^{2 m}<1\right\},
$$

where $m$ is a positive integer. Of course all the domains $\Omega_{m}$ are pseudoconvex and Reinhard.

However, as the following theorem shows, if we allow the boundary to be only $C^{1, \alpha}$-smooth at just one point, then the domain may be nonpseudoconvex and be non-equivalent to any Reinhardt domain.

Theorem 2. There exists a bounded, non-pseudoconvex domain $\Omega \subset \mathbb{C}^{2}$ with non-compact automorphism group and boundary smooth real-analytic everywhere except at one point (this exceptional point is an orbit accumulation point for the automorphism group action), and $C^{1, \alpha}$-smooth at the 
exceptional point for some $\alpha>0$, such that $\Omega$ is not biholomorphically equivalent to any Reinhardt domain.

For the proof of Theorem 2, we first need the following lemma, which is also of independent interest.

Lemma A. If $\Omega \subset \mathbb{C}^{2}$ is a bounded, non-pseudoconvex, simply-connected domain such that the identity component $\operatorname{Aut}_{0}(\Omega)$ of the automorphism group $\operatorname{Aut}(\Omega)$ is non-compact, then $\Omega$ is not biholomorphically equivalent to any Reinhardt domain.

Proof of Lemma A. Suppose that $\Omega$ is biholomorphically equivalent to a Reinhardt domain $D$. Since $\Omega$ is bounded, it follows that $D$ is hyperbolic. Also, since $\operatorname{Aut}_{0}(\Omega)$ is non-compact, then so is $\operatorname{Aut}_{0}(D)$. We are now going to show that any such domain $D$ is either pseudoconvex, or not simply-connected, or cannot be biholomorphically equivalent to a bounded domain. This result clearly implies the lemma.

We can now assume that the domain $D$ is written in its normalized form $\tilde{D}$ as in $(3)$, and $\operatorname{Aut}_{0}(\tilde{D})$ is given by formulas (2). Then, $\operatorname{since} \operatorname{Aut}_{0}(\tilde{D})$ is non-compact, it must be that $t>0$. Next, if $p=t$, then $\tilde{D}$ is either nonhyperbolic (for $s<t$ ), or (for $s=t$ ) is the unit ball or the unit polydisc and therefore is pseudoconvex. Thus we can assume that $t=1, p=2$, $n_{1}=n_{2}=1$.

Let $\tilde{D}_{1} \subset \mathbb{C}$ be the hyperbolic Reinhardt domain analogous to $\tilde{G}_{1}$ that was defined above (see (3)). Clearly, there are the following possibilities for $\tilde{D}_{1}$ :

(i) $\tilde{D}_{1}=\left\{0<\left|z_{2}\right|<R\right\}, 0<R<\infty$;

(ii) $\tilde{D}_{1}=\left\{r<\left|z_{2}\right|<R\right\}, 0<r<R \leq \infty$;

(iii) $\tilde{D}_{1}=\left\{\left|z_{2}\right|<R\right\}, 0<R<\infty$.

For the cases (i), (ii), $\tilde{D}$ is always not simply-connected, and therefore we will concentrate on the case (iii). If $s=0$, then $\tilde{D}$ is not hyperbolic since it contains the complex line $\left\{z_{2}=0\right\}$. Thus we can assume that $s=1$. Next observe that, for $\alpha_{1}^{2} \geq 0$, the domain $\tilde{D}$ is always pseudoconvex. Thus we may take $\alpha_{1}^{2}<0$. Then the domain $\tilde{D}$ has the form

$$
\tilde{D}=\left\{\left|z_{1}\right|<1,\left|z_{2}\right|<\frac{R}{\left(1-\left|z_{1}\right|^{2}\right)^{\gamma}}\right\}, \quad \gamma>0
$$

We will now show that the above domain $\tilde{D}$ cannot be biholomorphically equivalent to a bounded domain. More precisely, we will show that any bounded holomorphic function on $\tilde{D}$ is independent of $z_{2}$. 
Let $f\left(z_{1}, z_{2}\right)$ be holomorphic on $\tilde{D}$ and $|f|<M$ for some $M>0$. For every $\rho$ such that $|\rho| \leq \frac{R}{2}$, the disc $\Delta_{\rho}=\left\{\left|z_{1}\right|<1, z_{2}=\rho\right\}$ is contained in $\tilde{D}$. We will show that $\partial f / \partial z_{2} \equiv 0$ on every such $\Delta_{\rho}$, which implies that $\partial f / \partial z_{2} \equiv 0$ everywhere in $\tilde{D}$.

Fix a point $(\mu, \rho) \in \Delta_{\rho}$ and restrict $f$ to the disc $\Delta_{\mu}^{\prime}=\left\{z_{1}=\mu,\left|z_{2}\right|<\right.$ $R_{\mu}$ \}, where $R_{\mu}=R / 2\left(1-|\mu|^{2}\right)^{\gamma}$. Clearly, $(\mu, \rho) \in \Delta_{\mu}^{\prime}$ and $\overline{\Delta_{\mu}^{\prime}} \subset \tilde{D}$. By the Cauchy Integral Formula

$$
f\left(\mu, z_{2}\right)=\frac{1}{2 \pi i} \int_{\partial \Delta_{\mu}^{\prime}} \frac{f(\mu, \zeta)}{\zeta-z_{2}} d \zeta
$$

for $\left|z_{2}\right|<R_{\mu}$, and therefore

$$
\frac{\partial f}{\partial z_{2}}(\mu, \rho)=\frac{1}{2 \pi i} \int_{\partial \Delta_{\mu}^{\prime}} \frac{f(\mu, \zeta)}{(\zeta-\rho)^{2}} d \zeta
$$

Hence

$$
\left|\frac{\partial f}{\partial z_{2}}(\mu, \rho)\right| \leq \frac{M R_{\mu}}{\left(R_{\mu}-|\rho|\right)^{2}}
$$

Letting $|\mu| \rightarrow 1$ and taking into account that $R_{\mu} \rightarrow \infty$, we see that $\left|\partial f / \partial z_{2}(\mu, \rho)\right| \rightarrow 0$ as $|\mu| \rightarrow 1$. Therefore, $\partial f / \partial z_{2} \equiv 0$ on $\Delta_{\rho}$.

The lemma is proved.

Proof of Theorem 2. We will now present a domain that satisfies the conditions of the lemma. Consider first the following domain

$$
\Omega^{\prime}=\left\{\operatorname{Re} z_{1}+\frac{25 \sqrt{5}}{361}\left|z_{2}\right|^{38}+\left|z_{2}\right|^{18}-\left|z_{2}\right|^{10}+\left|z_{2}\right|^{2}<0\right\} .
$$

The domain $\Omega^{\prime}$ is clearly simply-connected, and $\partial \Omega^{\prime}$ is smooth real-analytic. Next, $\operatorname{Aut}_{0}\left(\Omega^{\prime}\right)$ is non-compact since it contains the subgroup $\left(z_{1}, z_{2}\right) \mapsto$ $\left(z_{1}+i t, z_{2}\right), t \in \mathbb{R}$. Further, $\Omega^{\prime}$ is non-pseudoconvex, as the Levi form of $\partial \Omega^{\prime}$ at every point $\left(z_{1}, z_{2}\right) \in \partial \Omega^{\prime}$ where $z_{2}=\frac{1}{\sqrt[8]{5}}$, is equal to $-\frac{18}{25}\left|z_{2}\right|^{2}$ and thus is negative-definite.

For the domain $\Omega$, we take the following bounded realization of $\Omega^{\prime}$. Namely, the mapping

$$
z_{1}^{*}=\frac{1}{z_{1}-1}, \quad z_{2}^{*}=\frac{z_{2}}{\left(z_{1}-1\right)^{\frac{1}{19}}}
$$


transforms $\Omega^{\prime}$ into the bounded domain

$$
\begin{aligned}
\Omega=\left\{\operatorname{Re} z_{1}^{*}+\left|z_{1}^{*}\right|^{2}\right. & +\frac{25 \sqrt{5}}{361}\left|z_{2}^{*}\right|^{38}+\left|z_{2}^{*}\right|^{18}\left|z_{1}^{*}\right|^{2-\frac{18}{19}} \\
& \left.-\left|z_{2}^{*}\right|^{10}\left|z_{1}^{*}\right|^{2-\frac{10}{19}}+\left|z_{2}^{*}\right|^{2}\left|z_{1}^{*}\right|^{2-\frac{2}{19}}<0\right\} .
\end{aligned}
$$

Since $\Omega$ is bounded, simply connected, non-pseudoconvex, and $\operatorname{Aut}_{0}(\Omega)$ is non-compact, Lemma $\mathrm{A}$ implies that $\Omega$ is not biholomorphically equivalent to any Reinhardt domain.

Next, it is easy to see that $\partial \Omega$ is smooth real-analytic everywhere except at $(0,0)$, and that it is of the class $C^{1, \frac{1}{19}}$ at $(0,0)$.

The theorem is proved.

Remarks.

1. The hypothesis of simple connectedness in Lemma A is automatically satisfied if, for example, the boundary of the domain is locally varietyfree and smooth near some orbit accumulation point for the automorphism group of the domain (see e.g. [GK2]). For a smoothly bounded domain this particular hypothesis would follow from a conjecture of Greene/Krantz [GK3].

2. One can also construct an example as in Theorem 2, where the domain $\Omega$ is pseudoconvex.

Let $\mathfrak{M}$ be the set of all subharmonic non-harmonic real-valued polynomials $P\left(z_{2}\right)$ on $\mathbb{C}$. Following $[\mathrm{O}]$, we introduce an equivalence relation on $\mathfrak{M}$. We say that $P_{1}, P_{2} \in \mathfrak{M}$ are equivalent, if there is a real number $\rho>0$, a holomorphic polynomial $p\left(z_{2}\right)$ and an automorphism $g\left(z_{2}\right)$ of $\mathbb{C}$ such that

$$
P_{1}\left(z_{2}\right)=\rho \operatorname{Re}\left(p\left(z_{2}\right)\right)+\rho P_{2}\left(g\left(z_{2}\right)\right) .
$$

Let $P\left(z_{2}\right) \in \mathfrak{M}$, and $\Omega^{\prime}$ be the domain.

$$
\Omega^{\prime}=\left\{\operatorname{Re} z_{1}+P\left(z_{2}\right)<0\right\} .
$$

The domain $\Omega^{\prime}$ is hyperbolic [BC]. Suppose now that $\Omega^{\prime}$ is biholomorphically equivalent to a Reinhardt domain $D$. Since $D$ also has to be hyperbolic and $\operatorname{Aut}_{0}(D)$ is non-compact, it follows from the proof of Lemma $\mathrm{A}$, that $D$ is either homogeneous, or $\operatorname{dim} \operatorname{Aut}(D)=4$. Then $[\mathrm{O}]$ implies that $\Omega^{\prime}$ is either biholomorphically equivalent to the unit ball (in which case $P\left(z_{2}\right)$ is equivalent to $\left|z_{2}\right|^{2}$ ), or to a complex ellipsoid $\Omega_{m}$, where $m \geq 2$ (in which case $P\left(z_{2}\right)$ is equivalent to $\left.\left|z_{2}\right|^{2 m}\right)$.

Therefore, to construct a domain $\Omega^{\prime}$ of the form (4) which is pseudoconvex, and is not biholomorphically equivalent to any Reinhardt domain, it 
suffices to choose $P\left(z_{2}\right) \in \mathfrak{M}$ in such a way that $P\left(z_{2}\right)$ is not equivalent to $\left|z_{2}\right|^{2 m}$ for any $m \in \mathbb{N}$. An example of a polynomial satisfying the above conditions is $P\left(z_{2}\right)=\left|z_{2}\right|^{2}+\left|z_{2}\right|^{2 m}$, where $m \geq 3$. Note that in this case the corresponding domain $\Omega^{\prime}$ has a bounded realization with boundary smooth real-analytic everywhere except at one point, and at the exceptional point the boundary is $C^{1, \alpha}$-smooth [BP1]. Indeed, by the mapping

$$
z_{1}^{*}=\frac{1}{z_{1}-1}, \quad z_{2}^{*}=\frac{z_{2}}{\left(z_{1}-1\right)^{\frac{1}{m}}}
$$

the domain

$$
\Omega^{\prime}=\left\{\operatorname{Re} z_{1}+\left|z_{2}\right|^{2}+\left|z_{2}\right|^{2 m}<0\right\}
$$

is transformed into the bounded domain

$$
\Omega=\left\{\operatorname{Re} z_{1}^{*}+\left|z_{1}^{*}\right|^{2}+\left|z_{1}^{*}\right|^{2\left(1-\frac{1}{m}\right)}\left|z_{2}^{*}\right|^{2}+\left|z_{2}^{*}\right|^{2 m}<0\right\}
$$

and the boundary of $\Omega$ is $C^{1, \frac{m-2}{m}}$-smooth at the exceptional point $(0,0)$.

It would be interesting to know if there exist analogous examples with a better regularity of the boundary at the exceptional point. A plausible conjecture seems to be that if $\partial \Omega$ is globally $C^{2}$ then the sort of pathology exhibited by the example in Theorem 2 cannot occur.

\section{References}

[BC] F. Berteloot and G. Cœuré, Domaines de $\mathbb{C}^{2}$, pseudoconvex et de type fini ayant un groupe non compact d'automorphismes, Ann. Inst. Fourier (Grenoble) 41 (1991), 77-88.

[BL] S. Bell and E. Ligocka, A simplification and extension of Fefferman's theorem on biholomorphic mappings, Invent. Math. 57 (1980), 283-289.

[BP1] E. Bedford and S. Pinchuk, Domains in $\mathbb{C}^{2}$ with non-compact holomorphic automorphism group (translated from Russian), Math. USSR-Sb. 63 (1989), $141-151$.

[BP2] Domains in $\mathbb{C}^{n+1}$ with non-compact automorphism groups, J. Geom. Anal. 1 (1991), 165-191.

[BP3] S., Convex domains with non-compact automorphism group (translated from Russian), Russian Acad. Sci. Sb. Math. 82 (1995), 1-20.

[C] H. Cartan, Les transformations analytiques des domaines cerclés les uns dans les autres, C. R. Acad. Sci. Paris 190 (1930), 718-720.

[GK1] R. E. Greene and S. G. Krantz, Characterization of certain weakly pseudoconvex domains with non-compact automorphism groups, Lecture Notes in Math. 1268, Springer-Verlag, 1987, 121-157.

[GK2] Invariants of Bergman geometry and the automorphism groups of domains in $\mathbb{C}^{n}$, Proceedings of a Conference on Complex Analysis and Geometry held in Cetraro, 1989, Mediterranean Press, 1992, 107-136. 
[GK3] - Techniques for studying automorphisms of weakly pseudoconvex domains, in J. E. Fornaess (Ed.) Several Complex Variables: Proceedings of the Mittag-Leffler Institute, 1987-1988, Math. Notes, Vol. 38, Princeton University Press, 1993, 389-410.

[Kob] S. Kobayashi, Intrinsic distances, measures and geometric function theory, Bull. Amer. Math. Soc. 82 (1976), 357-416.

[Kod] A. Kodama, A characterization of certain domains with good boundary points in the sense of Greene-Krantz, Kodai. Math. J. 12 (1989), 257-269.

[Kra] S. G. Krantz, Convexity in complex analysis, Several Complex Variables and Complex Geometry, Part 1 (Santa Cruz, CA, 1989), Proc. Sympos. Pure Math. 52 (1991), 119-137.

[Kru] N. G. Kruzhilin, Holomorphic automorphisms of hyperbolic Reinhardt domains (translated from Russian), Math. USSR-Izv. 32 (1989), 15-38.

[O] K. Oeljeklaus, On the automorphism group of certain hyperbolic domains in $\mathbb{C}^{2}$, Astérisque 217 (1993), 193-216.

[R] J. P. Rosay, Sur une caractérisation de la boule parmi les domaines de $\mathbb{C}^{n}$ par son groupe d'automorphismes, Ann. Inst. Fourier (Grenoble) 29 (1979), 91-97.

[W] B. Wong, Characterization of the unit ball in $\mathbb{C}^{n}$ by its automorphism group, Invent. Math. 41 (1977), 253-257.

Department of M athematics, Univerity of California, Riveride, Ca 92521 USA

E-mail address: sfu@math.ucr.edu

Centre for Mathematics and Its Applications, The Australian National University, C AnBerra, A C T 0200 A U S T R A LiA

E-mail address: Alexander.Isaev@anu.edu.au

Bergische Univerität, Gesamthochichule W uppertal, Mathematik (FB 07 ), Gaussitrasse 20, 42097 W uppertal, GERM A N

E-mail address: Alexander.Isaev@math.uni-wuppertal.de

Department of M athematics, Washington Univeritit, St.Louis, M O 63130 $\mathrm{USA}$

E-mail address: sk@math.wustl.edu

M SRI, 1000 Centennial D Rive, Berkeley, CA 94720 USA

E-mail address: krantz@msri.org 